\title{
Phase Contrast Images of Superconducting Pancake Vortices
}

\author{
M. Beleggia ${ }^{1,2}$, G. Pozzi ${ }^{1}$ and A. Tonomura ${ }^{3}$ \\ ${ }^{1}$ Dept. of Physics and INFM, University of Bologna, viale B.Pichat 6/2, 40127 Bologna, Italy. \\ 2 Now at: Dept. of Materials Science,Brookhaven National Laboratory, Upton, NY 11973, USA. \\ 3 Advanced Research Laboratory, Hitachi Ltd., Hatoyama, Saitama 350-0395, Japan.
}

The calculation of the electron optical phase shift experienced by high energy electrons in a transmission electron microscope, when they inte ract with the magnetic field associated with superconducting fluxons in a thin specimen tilted with respect to the beam is of fundamental importance for the interpretation of the experimental images. To perform this calculation, a new approach based on Fou rier space has been introduced [1]. The vector potential of the fluxon magnetic field is decomposed into its Fourier components and the phase shift of each component calculated separately and as a result, we obtained the Fourier transform of the phase, whi ch can be Fourier inverted, analytically or numerically, to recover the phase shift.

A considerable benefit of this new approach is that it can be profitably extended to cope with situations which could only be treated approximately, or not be dealt with at all, in the previous real space framework [2], like the case of pancake vortices in layered high $-T_{c}$ superconductors. In our model [1], the specimen of total thickness $t$ is divided into $n_{L}$ thin layers. Each layer is characterized by a Pearl penetration d epth $\Lambda=n_{L} \quad \lambda_{\mathrm{ab}}{ }^{2} / t$, where $\lambda_{\mathrm{ab}}$ is the transverse penetration depth characterizing the bulk material. The separation between the layers is therefore $s=t /\left(n_{L}-1\right)$. In order to have our model fairly representative of a real specimen the number of layers has bee $\mathrm{n}$ increased from 3 to 9, which is at the limit of our present computing facilities.

The case of a vortex pinned to an inclined columnar defect at $60^{\circ}$ and piercing the whole specimen is considered and the dependence of the out -of-focus contrast on the set -up geometry investigated. The obtained results are compared with those expected from the isotropic model in order to ascertain if anisotropy effects can be detected by the out-of-focus method.

Figure 1 reports an out -of-focus series calculated for two def ocus values, $200 \mathrm{~mm}$ (a) and $500 \mathrm{~mm}$ (b). The fluxon is in a specimen of thickness $400 \mathrm{~nm}$ tilted at $30^{\circ}$ with respect to the electron beam. The material is assumed to have a transverse penetration depth of $200 \mathrm{~nm}$. The first column displays the images obtained when the columnar defect is aligned with the projection of the normal to the specimen on the object plane, the second with a rotation of the specimen around its normal of $45^{\circ}$ and the third with a rotation of $90^{\circ}$. Comparison of the results obtained in the third column with the others confirms that this case represents the most favorable condition to detect the pinning of the fluxon core at the defect.

These same conditions have been used as input data for the isotropic model [3], and the obtained results a re reported in Fig. 2. The most striking difference is that in this case the pinning is more easily detectable and that the contrast increases with rotation angle, instead of decreasing as observed for the pancake model. This result is understandable because, for the chosen geometry, the Aharonov-Bohm effect across the tubes becomes more influential in affecting the image features. 
Therefore, these results indicate that not only pinning, but also the anisotropy of the fluxons can be detected by out -of-focus experiments if the experimental conditions are suitably chosen. This has been confirmed experimentally by the recent observations carried out with the new $1 \quad$-MV field emission electron microscope $[4,5]$.

\section{References}

[1] M. Beleggia and G. Pozzi, Ultramicroscopy 84, 171 (2000); Phys. Rev. B 63, 054507 (2001).

[2] S. Fanesi, et. al., Phys. Rev. B 59, 1426 (1999).

[3] R.G. Mints, et. al., Phys. Rev. B 61, 1623 (2000).

[4] A. Tonomura, et. al., Nature 412, 620 (2001).

[5] A. Tonomura, et. al., these Proceedings (2002).
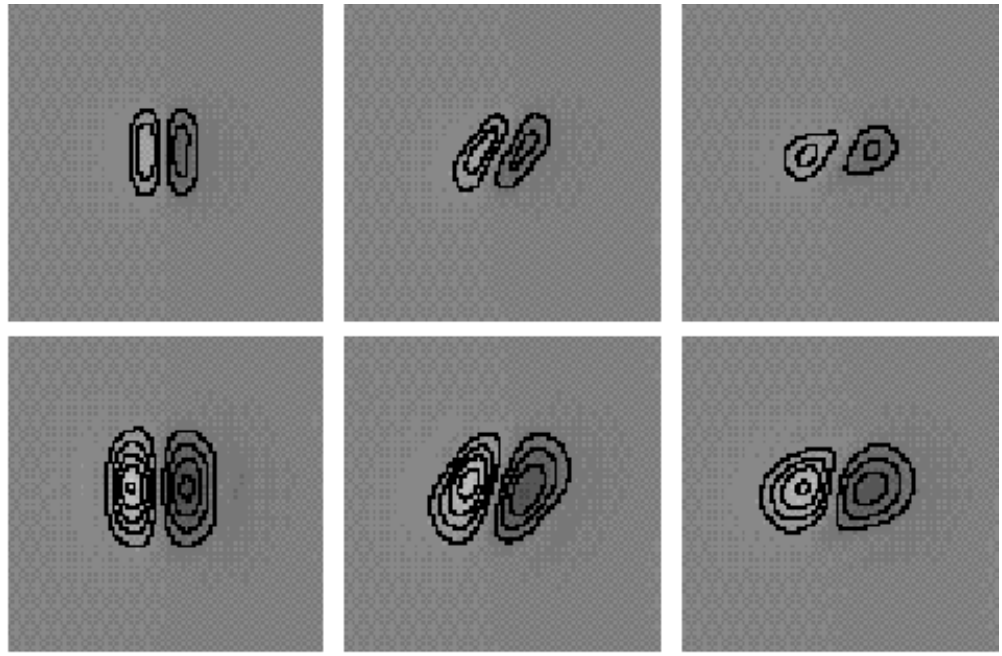

FIG. 1. Out of focus images of pancake vortices calculated for two different values of the defocus distance (from top to bottom: $200 \mathrm{~mm}$ and $500 \mathrm{~mm}$ ) and for different specimen rotation angles (from left to right: $0^{\circ}, 45^{\circ}, 90^{\circ}$ ). The square size of each image is $4 \mu \mathrm{m}$.
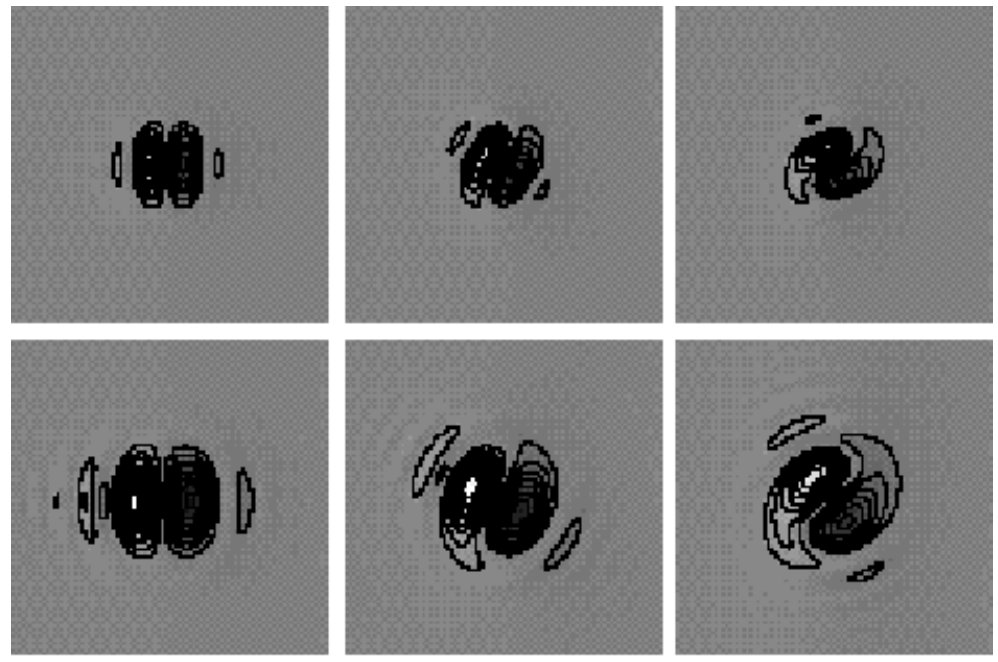

FIG. 2. Out of focus images of vortices in isotropic material calculated for two different values of the defocus distance (from top to bottom: $200 \mathrm{~mm}$ and $500 \mathrm{~mm}$ ) and for different specimen rotation angles (from left to right: $0^{\circ}, 45^{\circ}, 90^{\circ}$ ). The square size of each image is $4 \mu \mathrm{m}$. 